\title{
Quaderni
}

QUADERNI Communication, technologies, pouvoir

68 | Hiver 2008-2009

Militantisme médical et fabrique des politiques de santé

\section{Regards croisés dans la salle d'attente d'un hôpital : un lieu de fabrication des connaissances durables}

Hannah Gilbert

\section{(2) OpenEdition}

\section{Journals}

Édition électronique

URL : http://journals.openedition.org/quaderni/295

DOI : 10.4000/quaderni.295

ISSN : 2105-2956

Éditeur

Les éditions de la Maison des sciences de l'Homme

Édition imprimée

Date de publication : 5 janvier 2009

Pagination : 91-94

\section{Référence électronique}

Hannah Gilbert, «Regards croisés dans la salle d'attente d'un hôpital : un lieu de fabrication des

connaissances durables », Quaderni [En ligne], 68 | Hiver 2008-2009, mis en ligne le 05 janvier 2012, consulté le 01 mai 2019. URL : http://journals.openedition.org/quaderni/295 ; DOI : 10.4000/

quaderni.295 
regards croisés dans la salle d'attente d'un hôpital : un lieu de fabrication des connaissances durables

\section{Hannah} Gilbert

doctorante Social Studies of Medecine McGill University
Durant ces dernières années, les mesures publiques concernant la gestion sociale du sida se sont principalement orientées vers des programmes privilégiant un large accès aux thérapeutiques antirétrovirales (HAART). Le Sénégal est exemplaire à ce propos puisque en 1988, ce pays est le premier à bénéficier d'une telle initiative de la part du gouvernement africain (Ndoye et al. 2002). Dans ce contexte, les patients profitant de la thérapie antirétrovirale (ARV) doivent scrupuleusement respecter la fréquence de leur médication afin de supprimer la présence virale et de freiner la survenue de résistances. À partir d'observations ethnographiques portant sur les relations informelles entre patients traités, l'objectif de cette note de recherche consiste à décrire la manière dont les connaissances théoriques relatives aux virus et à leurs thérapeutiques sont traduites en données pratiques. En partant d'une observation sur le terrain, nous allons décrire la manière dont une forme de standardisation informelle s'affirme progressivement dans le maniement collectif de la médication, avec pour effet, de faciliter l'adhésion à ces protocoles.

Une femme, Astou', est installée sur l'une des chaises en plastique alignées le long du mur de la salle d'attente silencieuse, en attente de recevoir son premier traitement antivirale. Le bâtiment comporte seulement une salle d'examen, un bureau de travailleurs sociaux rarement occupé et une aire de stockage hospitalière laissée à l'abandon. Une autre jeune femme, Bintou, marche le long de la salle puis s'installe sur la chaise avec la même ferme intention d'arriver en avance à son rendez-vous médical. Les deux femmes rentrent facilement en conversation et Bintou est très rapidement disposée à aider Astou 
pour sa première visite. Tandis qu'elles visitent ensemble l'hôpital, Bintou expose à Astou son récit de mise en route du traitement. Par la suite, elle prend Astou à part après chaque rendez-vous médical et elle lui donne des instructions complémentaires aux consultations officielles sur la manière de prendre la médication antirétrovirale en s'appuyant sur sa propre expérience de patient traité. Elle aide également son interlocutrice à faire comprendre ses besoins auprès de l'équipe hospitalière. En particulier, Bintou discerne la difficulté qu'éprouve Astou à lire et à écrire. Lorsque les deux femmes rencontrent le pharmacien, Bintou lui suggère de tracer des cercles sur chacun des flacons de médicament antiviral pour que Astou puisse se souvenir de son régime médicamenteux. En fait, Astou craint d'utiliser les services officiels du programme de peur de rencontrer des personnes qu'elle connaît, aussi, elle voit dans ces échanges informels, un moyen efficace pour adhérer au protocole antiviral.

Le programme-pilote assure mensuellement une dose de médicaments à chaque patient. Ainsi, un mois plus tard, lorsque Astou arrive à l'hôpital pour prendre sa deuxième dose, elle retrouve Bintou installée dans la salle d'attente, au même endroit que lors de la première rencontre. La régularité de ces échanges entre patients est à la base de la constitution de savoirs implicites. Ces réunions informelles, en faisant ressortir la réalité concrète hétérogène des modalités d'applications du programme médicamenteux, constituent un point central dans la production et la transmission des connaissances sur la manière d'adhérer au traitement antivirale. L'observation ethnographique révèle que les échanges informels se déroulant dans la salle d'attente forment un terreau fondamental à l'acquisition et à la stabilisation des connaissances relatives à l'adhésion aux protocoles antiviraux. Les médecins et les travailleurs sociaux impliqués dans le programme fournissent des informations faisant écho aux standards cliniques. Comme le révèle l'expérience d'Astou à l'hôpital, les relations informelles entre les patients apposent un filtre à ce discours officiel. Ces échanges informels sont intimement reliés à des objets courants et à des pratiques profanes, traditionnellement considérés comme extérieurs au déroulement du traitement antiviral. Le principe de symétrie fournit un cadre analytique permettant de comprendre comment ce large assemblage de personnes et d'objets constitue un réseau de connaissances sur l'adhésion au traitement (Callon et Latour 1981, Callon 1986, Latour 1983, 1988). Par exemple, les empilements de châssis de lits rouillés qui jointent l'emplacement de la salle d'attente effectuent une contrainte, ils laissent la place à un seul espace retreint au sein du petit pavillon où les médecins administrent la thérapie antivirale. La disposition de cette salle d'attente privée, inhabituelle dans un contexte de surpeuplement des hôpitaux publics, laisse un espace calme à Astou et à son amie au sein duquel elles peuvent échanger les détails personnels de leurs expériences de traitements.

Si les châssis de lits rouillés et les chaises en plastique interviennent de façon déterminant dans la constitution des connaissances qui émergent de cette situation locale, ces objets physiques ne renvoient pas à une représentation commune et stabilisée pour les acteurs en présence (Pickering 1999). Pour bien comprendre ce point, il est important de resituer ce réseau de relations de patients dans l'environnement culturel et 
historique particulier afin de contextualiser ce type d'agencement et de comprendre comment les disparités entre ces éléments apportent des différences dans la manière de former le réseau (Strathern 1999:157, de Laet et Mol 2000). Ainsi, l'établissement de soutien entre les patients et les débris hospitaliers ne sont pas derechef intimement liés. Le rôle primordial joué par ces derniers matériaux dans la production des connaissances sur la thérapie antivirale provient de la convergence d'aspects historiques, culturels et économiques du quotidien de vie dans un contexte post-colonial (Anderson 2002). Dans un pays à faible ressource comme le Sénégal, de nombreuses femmes comme Astou dépendent exclusivement des relations familiales ou communautaires pour leur subsistance. Nombreuses parmi celles qui vivent avec le sida ont stratégiquement maintenu ce type de connexions en cachant leur statut sérologique par le fait que la connaissance de leur infection pourrait compromettre leur accès aux soins. L'introduction de la thérapie antivirale a augmenté le désir pour ces femmes de maintenir les relations avec d'autres patients afin de faciliter le partage des connaissances concernant cette thérapeutique. Toutefois, certains patients expriment une réticence à s'engager dans un groupe officiel de soutien parce qu'ils sentent que cette position compromet leur aptitude à jauger les autres patients avant de partager leur statut sérologique. Dans ce contexte, la présence de la salle d'attente, délimitée par le tracé spatial des déchets hospitaliers encourage les échanges entre patients ainsi que les formes émergentes locales de connaissances relatives à la thérapie antivirale.

Les anthropologistes ont démontré la nature so- cio-culturelle du travail scientifique en examinant comment de nouvelles formes d'association ont émergé autour des nouvelles technologies médicales (i.e. Rabinow 1996, Rapp 2000, Nguyen 2002). Tout comme d'autres formes institutionnalisées de connaissances, les données concernant l'adhésion à la thérapie antivirale sont exprimées à travers un contexte de relations sociales. En suivant la trajectoire des patientes, cette narration met en évidence une ressource possible à partir de laquelle les patients modèlent et re-forment ce type de savoir en une modalité pratique correspondant aux nécessités locales. Bien entendu, l'expérience du patient inséré dans la relation clinique demeure un axe d'étude indispensable pour suivre la prolifération officielle des connaissances concernant l'adhérence, de même que les laboratoires et les arènes politiques constituent des pivots à l'analyse des nouvelles formes de socialité émergeant autour des antiviraux dans un contexte post-colonial. Cependant, comme nous avons tenté de le montrer, le déplacement des recherches ethnographiques vers ces nouvelles arènes repositionne également le curseur théorique, il permet de rediscuter l'idée courante selon laquelle la notion de circulation des connaissances biomédicales et des technologies implique la colonisation successive de la diversité locale. De manière probante, l'observation d'une salle d'attente hospitalière montre comment les négociations autour des formes locales de connaissances participent à asseoir et à fortifier le savoir biomédical global. 
$R \cdot E \cdot F \cdot E \cdot R \cdot E \cdot N \cdot C \cdot E \cdot S$

ANDERSON, W., "Postcolonial technoscience », 2002, Social Studies of Science 32(5-6): 643-658.

CALLON, M., « Some elements of a sociology of translation: Domestication of the scallops and the fishermen of Saint Brieuc Bay » in J. Law (ed.) Power, Action and Belief : A New Sociology of Knowledge? Sociological Review Monograph, 1986, London : Routledge and Kegan Paul. 32: 196-233.

CALLON, M., LATOUR, B., « Unscrewing the Big Leviathan : how actors macrostructure reality and how sociologists help them to do so », 1981, in K. D. Knorr-Cetina and A. V. Cicourel (eds.) Advances in Social Theory and Methodology : Toward an Integration of Micro- and Macro-Sociologies. Boston, Mass, Routledge and Kegan Paul : 277-303.

De LAET, M., MOL A., « The Zimbabwe Bush Pump: Mechanics of a fluid technology », 2000, in Social Studies of Science: 30(2):225-63.

LATOUR, B., « Give me a laboratory and I will raise the world », 1983, in K. Knorr-Cetina and M. Mulkay (eds) Science Observed, London : Sage : 141-170. The Pasteurization of France, 1988, Cambridge : Mass : Harvard University Press.

NDOYE, I., et al., « Présentation de l'initiative sénégalaise d'accès aux antirétroviraux », 2002, in A. Desclaux et al. (eds) L'Initiative Sénégalaise d'Accès aux Médicaments Antirétroviraux, Paris : ANRS, Éditions EDK.

NGUYEN, V.-K., «Sida, ONG et la politique du témoignage en Afrique de l'Ouest », 2002, in Anthropologie et Sociétés 26(1): 69-87.
PICKERING, A., " The mangle of practice. Agency and emergence in the sociology of science », 1999, in M. Biagioli (ed.) The science studies reader. New York : Routledge.

RABINOW, P., «Artificiality and Enlightenment : from sociobiology to biosociality », 1996, in Essays on the Anthropology of Reason. Princeton, New Jersey : Princeton University Press.

RAPP, R., « Extra chromosomes and blue tulips: medico-familial conversations », 2000, in A. Cambrosio, M. Lock and A. Young (eds.) Living and Working with the New Medical Technologies. Cambridge: Cambridge University Press : 184-208.

STRATHERN, M., «What is intellectual property after ? », 1999, in J. Law and J. Hasard (eds.) Actor Network Theory and After. Oxford : Blackwell : 156-180.

1. Afin d'assurer l'anonymat des deux patientes et du personnel impliqué dans leurs soins, leurs identités dépeintes dans la narration s'appuient sur des personnages composés à cette fin. 